\title{
Systemic vasculitis and aneurysm formation in the Wiskott-Aldrich syndrome
}

\author{
W G McCluggage, D J Armstrong, R J Maxwell, P K Ellis, D R McCluskey
}

\begin{abstract}
A 24 year old male who suffered from the Wiskott-Aldrich syndrome developed intra-abdominal bleeding on two occasions. Radiological investigations showed aneurysmal dilatation of branches of the hepatic and superior mesenteric arteries. The second abdominal bleed necessitated laparotomy and the bleeding was localised to the kidneys. Right nephrectomy was performed and histological examination showed a necrotising vasculitis, mainly involving medium and small sized renal blood vessels. Steroids, immunosuppressive treatment, and control of blood pressure resulted in resolution of the vasculitic process and prevented further haemorrhage. Vasculitis and aneurysm formation are rarely described complications of Wiskott-Aldrich syndrome and may account for the life threatening haemorrhage which occurs in this condition. (f Clin Pathol 1999;52:390-392)
\end{abstract}

Keywords: kidney; vasculitis; aneurysm; Wiskott-Aldrich syndrome

The Wiskott-Aldrich syndrome is a rare $\mathrm{X}$ linked recessive disorder characterised by immune deficiency, eczema, and thrombocytopenia. ${ }^{12}$ The underlying defect has been identified as a deficiency of the WAS protein, a 502 amino acid protein involved in signal transduction via GTPase. ${ }^{3}$ Deficiency of the protein affects reorganisation of the actin cytoskeleton and also causes platelet function abnormalities. In severe cases, both humoral and cell mediated immunity are impaired, resulting in an increased susceptibility to infection. There is also an increased risk of lymphoid malignancies, especially nonHodgkin's lymphoma and acute myeloid leukaemia. ${ }^{4}$ The development of malignancy is usually associated with decreased immune surveillance and is confined to those patients with clinically significant immune deficiency. Renal involvement in the form of glomerulonephritis has been described in the Wiskott-Aldrich syndrome and various histological patterns may be seen, including membranoproliferative and mesangial glomerulonephritis. ${ }^{5}{ }^{6}$ IgA nephropathy has also been described, ${ }^{7}$ which may be secondary to immune complex deposition as a result of recurrent infections.

To date, only occasional cases of vasculitis or aneurysms have been reported in patients with the Wiskott-Aldrich syndrome, ${ }^{8-13}$ and the association is not well established. This report describes a patient with Wiskott-Aldrich syndrome in whom a florid necrotising vasculitis involved medium and small sized blood vessels, resulting in aneurysmal dilatation and complicated by rupture with massive retroperitoneal haemorrhage necessitating nephrectomy. Possible aetiological factors in the development of vasculitis in the Wiskott-Aldrich syndrome are discussed.

\section{Case report}

A 24 year old man had been under lifelong review for Wiskott-Aldrich syndrome. From infancy he suffered from recurrent upper and lower respiratory tract infections, resulting in bronchiectasis involving the lower lobe of the left lung. In the second decade of life he developed problems attributable to thrombocytopenia, with epistaxis and bruising. By his early twenties he had several haemarthroses, purpura, and severe bruising, and his platelet count had fallen to below 10 000/dl. Following pneumovax vaccination, splenectomy was performed and his platelet count returned to normal. However, three months later he was admitted to hospital with abdominal pain, melaena, and haematemesis. A small bowel series showed small intestinal obstruction and at laparotomy adhesions were divided.

Postoperative recovery was complicated by a chest infection and persistent hypertension and two weeks after laparotomy he suddenly developed severe abdominal pain and acute gastrointestinal haemorrhage. Computed tomography of the abdomen and arteriography showed multiple aneurysms arising from distal branches of the right and left hepatic arteries (fig 1). Fresh thrombus was seen around one of the left hepatic artery aneurysms suggesting this as the source of bleeding. Following

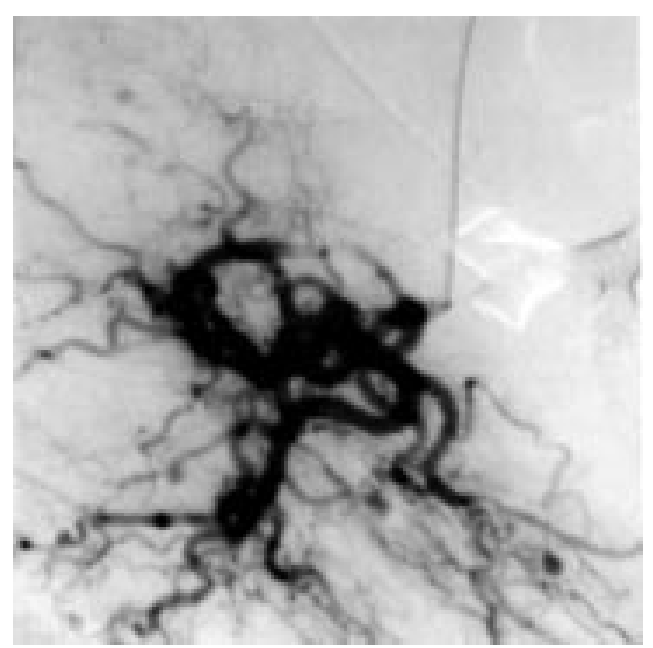

Figure 1 Arteriogram showing aneurysmal dilatation of hepatic blood vessels.
Accepted for publication 15 December 1998 
arteriography, distal and proximal embolisation of the left hepatic artery was performed and haemostasis was achieved.

He remained hypertensive with a low grade pyrexia, a leucocytosis, and a serum $\mathrm{C}$ reactive protein of $320 \mathrm{~g} /$ litre. Although a vasculitis screen, including P-ANCA and C-ANCA, was negative, the underlying cause of the aneurysms was thought to be vasculitis and steroid treatment was initiated. Within the next seven days he had a further acute episode of gastrointestinal bleeding. Repeat arteriography showed several aneurysms within the jejunal branches of the superior mesenteric artery and again distal embolisation was performed. His condition did not improve and a further laparotomy was performed, during which massive bilateral retroperitoneal haemorrhage was found. Haemostasis was achieved around the left kidney and intraoperative intravenous pyelography confirmed the viability of the kidney. Exposure of the right kidney revealed multiple capsular tears, all bleeding profusely, and nephrectomy was necessary to achieve haemostasis. He required multiple transfusions with whole blood, packed cells, fresh frozen plasma, and platelets.

Postoperatively the immunosuppressive treatment was increased with the addition of cyclophosphamide $100 \mathrm{mg}$ daily and prednisolone $40 \mathrm{mg}$ daily. The serum $\mathrm{C}$ reactive protein fell to $3.6 \mathrm{~g} /$ litre and the patient felt better. Antihypertensive treatment was increased to maintain a systolic blood pressure of less than $160 \mathrm{~mm} \mathrm{Hg}$. Seven weeks after nephrectomy he remains stable with normal serum urea and creatinine levels and with no further evidence of blood loss.

\section{Pathological findings}

The resected right kidney weighed $224 \mathrm{~g}$ with a bipolar length of $13 \mathrm{~cm}$. Several foci of fresh haemorrhage were seen on the cortical surface.

Histological examination showed a necrotising vasculitis involving medium and small sized arteries (fig 2A), mainly at the renal hilum but also within the substance of the kidney. The involved arteries contained eosinophilic fibrinoid material within their walls. This showed the characteristic staining properties of fibrin with the Martius scarlet blue (MSB) stain and was associated with an inflammatory cell infiltrate, chiefly consisting of lymphocytes, plasma cells, histiocytes, and neutrophils. Nuclear dust was prominent. Multinucleate giant cells were not identified. Several of the involved blood vessels showed marked fibrous intimal proliferation with almost total obliteration of the lumen (fig 2B), while others contained organising thrombus. The Weigarts' elastic tissue stain (WETS) showed marked destruction of elastic fibres within the walls of the affected arteries (fig 2C). Within the renal parenchyma, an area of organising haemorrhage was present. In the centre of this there was a dilated blood vessel which was involved by the necrotising vasculitis.

The renal glomeruli showed no evidence of a glomerulonephritic process.
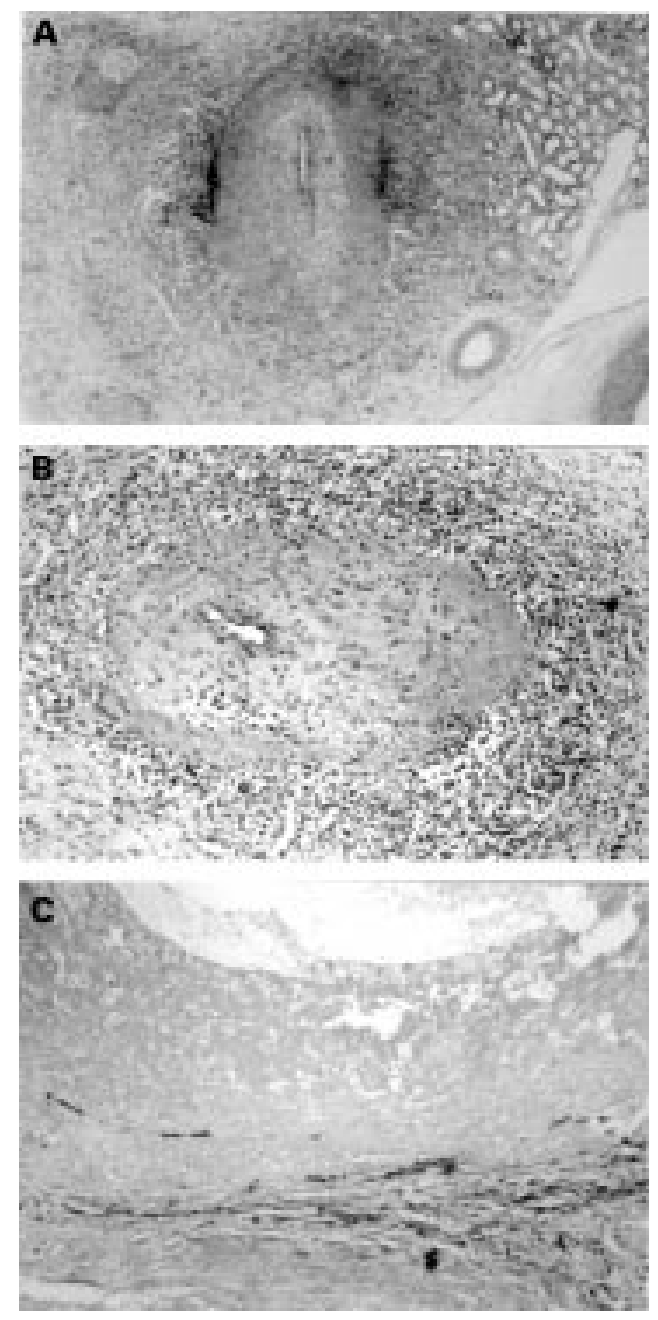

Figure 2 (A) Necrotising vasculitis involving medium sized artery at hilum of the kidney. (B) This artery, which is affected by vasculitis, shows marked fibrous intimal proliferation. (C) Weigarts'elastic tissue stain (WETS stain) showing destruction of elastic fibres within the wall of the artery.

\section{Discussion}

Occasional cases of vasculitis and aneurysms have been reported in the Wiskott-Aldrich syndrome, but the association is not well established. In this case, radiological studies indicated aneurysmal dilatation of blood vessels within the liver and the small intestine, and following nephrectomy a necrotising vasculitis was identified involving medium and small sized blood vessels within the kidney. We speculate that blood vessels within the liver and the small intestine were also involved by this vasculitic process and that the affected renal blood vessels underwent aneurysmal dilatation with subsequent rupture resulting in retroperitoneal haemorrhage. The histological features in the present case, as well as the distribution of the vasculitis, resemble those seen in polyarteritis nodosa. However, although serum C reactive protein was raised, serum P-ANCA was negative.

Lau et al reported a case of Takayasu's arteritis in a patient with the Wiskott-Aldrich syndrome. ${ }^{10}$ The diagnosis was based on the aortographic findings of an aortic aneurysm associated with bilateral stenosis of the renal 
arteries. There was no histological confirmation of a vasculitic process. A case of isolated arteritis involving cerebral blood vessels has also been reported, ${ }^{11}$ and Filipovich et al described a case of fatal arteritis with infarcts in the heart, liver, gallbladder, and stomach. ${ }^{12}$ In another case, where the diagnosis of vasculitis was made at necropsy, terminal massive retroperitoneal and subcapsular renal bleeding was present. ${ }^{13}$ In addition, a case of leucocytoclastic vasculitis has been described in a patient with the Wiskott-Aldrich syndrome. ${ }^{8}$ However, in this last case the patient also had a disseminated $\mathrm{T}$ cell lymphoma and it is possible that the vasculitis was related to the lymphoma. Ilowite et al described a case of pulmonary angiitis in a patient with the Wiskott-Aldrich syndrome. ${ }^{9}$ The histological features in this case were typical of lymphomatoid granulomatosis which is now thought to represent an angiocentric and angiodestructive malignant lymphoma and is not considered a true vasculitis.

It is unusual for patients with the WiskottAldrich syndrome to survive into adulthood. However, with recent therapeutic improvements and better management, affected individuals are surviving longer and it may be these patients who are most at risk of developing complications such as lymphoproliferative malignancy and vasculitis. Why patients with the Wiskott-Aldrich syndrome should be at risk of developing vasculitis is not clear. However, abnormalities of serum immunoglobulins are often present, characteristically increased IgA and IgE and depressed IgM. ${ }^{14}$ It is possible that deposition of immunoglobulins within vessel walls may be responsible for the development of vasculitis. Alternatively, such patients are susceptible to a variety of infections, and the vasculitis may be a manifestation of an abnormal immune reaction to an infecting organism.

In conclusion, we report a case of histologically confirmed vasculitis with resultant aneurysm formation in a patient with the WiskottAldrich syndrome. Although the two conditions may be coincidental, this case together with those previously described suggests an association. Further cases are likely to be encountered as patients with the
Wiskott-Aldrich syndrome survive longer. Individuals with the Wiskott-Aldrich syndrome who develop evidence of vasculitis such as abnormal renal function, hypertension, or a rise in ESR or C reactive protein should be investigated for possible aneurysmal dilatation of blood vessels, and immunosuppressive treatment considered. If hypertension is present this should be closely monitored and treated. The risk of death from aneurysmal rupture is increased in Wiskott-Aldrich syndrome owing to reduced platelet number and function. It is possible that vasculitis and aneurysm formation is a more common complication than previously recognised and this may account for the cases of reported deaths from cerebrovascular haemorrhage without marked thrombocytopenia.

1 Aldrich RA, Steinberg AG, Campbell DC. Pedigree demonstrating a sex-linked recessive condition characterised by draining ears, eczematoid dermatitis and bloody diarrhea. Pediatrics 1954;13:133-8.

2 Standen GR. Wiskott-Aldrich syndrome: a multidisciplinary disease. F Clin Pathol 1991;44:979-82.

3 Symons M, Derry JM, Karlak B, et al. Wiskott-Aldrich syndrome protein, a novel effector for the GTPase CDC $42 \mathrm{Hs}$, is implicated in actin polymerization. Cell 1996;84:723-34.

4 Perry GS, Spector BD, Schuman LM, et al. The Wiskott-Aldrich syndrome in the United States and Canada (1892-1979). F Pediatr 1980;97:72-8.

5 Gutenberger J, Trygstad CW, Stiehm ER, et al. Familial thrombocytopenia, elevated serum IgA levels and renal disease. A report of a kindred. Am $\mathcal{F}$ Med 1970;49:729-41.

6 Spitler LE, Wray BB, Mogerman S, et al. Nephropathy in the Wiskott-Aldrich syndrome. Pediatrics 1980;66:391-8.

7 DeSanto NG, Sessa A, Capodicasa G, et al. IgA glomeruDesanto NG, Sessa A, Capodicasa G, et al. IgA glomeru-
lonephritis in Wiskott-Aldrich syndrome. Child Nephrol Urol 1988;89:118-20.

8 Watson RD, Gershwin ME, Smithwick E, et al. Cutaneous T cell lymphoma and leukocytoclastic vasculitis in a longterm survivor of Wiskott-Aldrich syndrome. Ann Allergy 1985;55:654-7.

9 Ilowite NT, Fligner CL, Ochs HD, et al. Pulmonary angiitis with atypical lymphoreticular infiltrates in Wiskott-Aldrich syndrome: possible relationship of lymphomatoid granulomatosis and EBV infection. Clin Immunol Immunopathol 1986;41:479-84.

10 Lau YL, Wong SN, Lawton WM. Takayasu's arteritis associated with Wiskott-Aldrich syndrome. 7 Pediatr Child Health 1992;28:407-9.

11 Nozicka Z. Isolated arteritis in the basal cerebral arteries in a child with primary immunodeficiency due to the Wiskott-Aldrich syndrome. Cesk Patol 1987;23:215-21.

12 Filipovich AH, Krivit W, Kersey JH, et al. Fatal arteritis as a complication of Wiskott-Aldrich syndrome. F Pediatr 1979; 95:742-4.

13 Hitzig WH, Truniger B. Wiskott-Aldrich syndrome: a truly interdisciplinary problem. Nephrol Dial Transplant 1996;11: 2093-5.

14 Radl J, Dooren LJ, Morell A, et al. Immunoglobulins and transient paraproteins in sera of patients with the WiskottAldrich syndrome: a follow up study. Clin Exp Immunol 1976;25:256-63. 